\title{
Comparison of modified suture less peritoneal dialysis catheter placement with conventional technique: a prospective study
}

\begin{abstract}
Introduction: Continuous Ambulatory Peritoneal Dialysis (CAPD) is a type of dialysis that uses the peritoneum in a person's abdomen as the membrane through which fluid and dissolved substances are exchanged with the blood. It is used to remove excess fluid, correct electrolyte problems, and remove toxins in those with renal failure. We developed a new technique for percutaneous Continuous Ambulatory Peritoneal Dialysis (CAPD) catheter insertion. Our new technique, in addition to being easy, proved to be safe and less time consuming.
\end{abstract}

Aim: To compare and analyze the benefits of Modified method of percutaneous placement of peritoneal dialysis catheter with the Conventional technique.

Patients \& methods: This prospective study was conducted in the department of Nephrology at Government Medical College, Kota on 97 patients. Group A included 49 patients and Group B included 48 individuals. Patients who had percutaneous Tenckhoff catheters inserted both by Conventional method (Group A) and Modified method (Group B) were included in the study and analyzed, based on total duration of procedure, early complications and benefits of the modified technique.

Results: Data of 97 patients were analyzed. The mean age in group A was $51.65+18.66$ years and $51.79+16.60$ years in group B.

i. Early complications including CAPD fluid leak was found in $44.89 \%(22 / 49)$ in Conventional method and $4.17 \%(2 / 48)$ in modified method ( $\mathrm{p}$ value $<0.05)$.

ii. We found $23(46.93 \%)$ episodes of peritonitis in conventional group and $6(12.5 \%)$ episodes after modified percutaneous insertion ( $\mathrm{p}$ value $<0.05$ ). Twenty episodes $(41.67 \%)$ of catheter migration were observed in modified method and 3 episodes $(6.122 \%)$ in conventional method ( $\mathrm{p}$ value $<0.05)$ but with significantly better CAPD duration $(21.43+11.69 \mathrm{v} / \mathrm{s} 15.89+9.01)$ and significantly lesser incidences of drainage failure $(8.33 \% \mathrm{v} / \mathrm{s} 46.94 \%)(\mathrm{p}$ value $<0.05)$

iii. Duration of hospital stay was also less in modified method(3.62+1.23 days) as compared to conventional method $(4.89+0.99$ days $)(p$ value $<0.05)$. Late onset CAPD fluid leak and peritonitis were more in the conventional method.

Conclusion: Modified CAPD catheter insertion has lower early and late complication rates and lower incidences of drainage failure and shorter duration of hospital stay.

Keywords: peritoneal dialysis catheter, government medical college, kota, modified technique, CAPD

\author{
Volume 6 Issue I - 2018 \\ Vikash Khandelia,' Ashutosh sharma, ${ }^{2}$ Pawan \\ Kumar Pyarasabadi ${ }^{3}$ \\ 'Head department of nephrology, government medical college \\ Kota, India \\ ${ }^{2}$ Department of community medicine, government medical \\ college Kota, India \\ ${ }^{3}$ Department of medicine, Rajarajeswari medical college and \\ hospital, India
}

\section{Correspondence: Vikash Khandelia, head department of nephrology, government medical college, B 56, Near BSNL Circle, Shrinathpuram, Kota, Rajasthan, India, Tel 919928699529,} Email vikasnephro@gmail.com

Received: September 12, 2017 | Published: January 31, 2018
Abbreviations: CAPD, continuous ambulatory peritoneal dialysis; APD, automated peritoneal dialysis; CKD, chronic kidney disease; HD, hemodialysis; PD, peritoneal dialysis

\section{Introduction}

In renal failure, haemodialysis is the preferred method to correct the accumulation of toxins, electrolytes, and fluid. Peritoneal dialysis is less efficient than haemodialysis, but it is preferred in children, diabetic patients, and patients with unstable cardiovascular disease; it is also used in patients who can manage their condition, or those who live far from a dialysis centre. It is unsuitable for patients who have had significant abdominal surgery. In peritoneal dialysis, the solution is infused into the peritoneal cavity, where exchange of electrolytes takes place by diffusion and convection, and excess fluid is removed by osmosis, using the peritoneal membrane as an osmotic membrane. There are 2 forms of peritoneal dialysis:

1. Continuous ambulatory peritoneal dialysis (CAPD), in which dialysis is performed manually by the patient several times each day;

2. Automated peritoneal dialysis (APD), in which dialysis is performed by machine overnight.

The increasing prevalence of end stage renal disease over recent years due to better survival of chronic kidney disease(CKD) patients has led to an increased number of both dialysis access procedures, that is hemodialysis (HD) and Peritoneal Dialysis (PD) as well as extra workload for those involved to treat them. Continuous Ambulatory Peritoneal Dialysis (CAPD) is underused in clinical field, despite 
having several advantages compared with Hemodialysis (HD), which include the preservation of residual renal function, freedom from pain from fistula needle, better hemodynamic stability, better survival in the early dialysis period and better quality of life..$^{1-3}$ The success of peritoneal dialysis depends on a well-functioning peritoneal catheter. Use of best techniques in catheter insertion can minimize the risk of catheter complications and peritoneal dialysis failure. ${ }^{4}$

There are various methods to implant PD catheters, which include open method, laparoscopic method, the peritoneoscopic method, percutaneous trocar and cannula method or Seldinger method. Each method has its advantages and disadvantages. Despite the improved results with newer approaches, many institutions have suboptimal access to surgical PD catheter placement, problems related to operating room scheduling or other operational inefficiencies that can make surgical PD catheter placement impractical.

The advantages of percutaneous PD catheter insertion over surgical methods include lower catheter-related mechanical and infectious complication rates, higher long-term catheter patency and the absence of needs for surgery and general anesthesia. ${ }^{5,6}$ However, the percutaneous PD catheter insertion technique has not been universally accepted due to the early mechanical complications and the potential risk for bowel perforation associated with its blind puncture method. ${ }^{7,8}$ Hence, there have been several attempts to maximize the advantages of percutaneous PD catheter insertion, while minimizing its disadvantages. ${ }^{9}$

The availability of timely PD catheter insertion, low rate of complication related with procedure and the low rate of catheter failure with efforts of nephrologists are important requisites that will increase the utility of CAPD and reduce patient morbidity. ${ }^{7}$

The purpose of this study is to describe our modified percutaneous PD catheter insertion method, and to show its clinical advantages over conventional method.

\section{Materials and methods}

This prospective study includes patients who underwent percutaneous PD catheter insertion by nephrologist at government medical college, Kota, India between Jan 2014 and Dec 2015. After obtaining IRB approval, informed written consent was taken from all patients. A total of 97 patients underwent PD catheter insertion, 48 patients by modified method (group M) and 49 patients by conventional method (group A). Statistical differences between the groups were assessed using chi-square test for categorical data and Student's t-test for continuous data. A $p$ value $<0.05$ was regarded statistically significant.

The patients were evaluated for their baseline characteristics, including demographics, medical history, drug history, and laboratory examination. The complication rate, procedure time and hospitalization period were compared between group $\mathrm{M}$ and group A. Then, the complications were subcategorized into intra-operational bowel injury, PD fluid leakage, catheter tip migration, drainage failure and peritonitis to compare the complications in more detail.

\section{Inclusion criteria}

\section{Cases:}

1. Patients of kidney failure requiring dialysis support.

2. Willing to participate in study and give written consent.

\section{Exclusion criteria}

\section{Cases:}

1. History of abdominal surgery in past.

2. Who were not willing to participate in study?

3. Those patients who were seriously ill.

\section{Follow up:}

All patients were followed up for at least one year or more to compare the benefits of both methods.

\section{CAPD catheter insertion method}

All procedures were performed under local anesthesia. Prophylactic antibiotics were given intravenously to all patients. A double cuffed straight type Tenckhoff catheter was used in conventional group and curl type in modified group. Immediately after catheter insertion, peritoneal dialysis was performed to check any obstruction or bleeding in the outflow. Thereafter catheters were flushed regularly before commencement of regular dialysis.

PD catheter insertion method was performed as follows:

\section{Conventional method for PD catheter insertion}

Percutaneous Needle-Guide wire Technique Placement of catheters by percutaneous puncture without image guidance was performed using a modification of the Seldinger technique, under local anesthesia. With the patient in supine position, the abdomen was prefilled with 1.5 to $2 \mathrm{~L}$ of dialysis solution instilled with an 18-gauge introducer needle which was inserted through a 4 to $5 \mathrm{~cm}$ infraumbilical incision with blunt dissection of subcutaneous fat down to the linea alba. Thereafter, a guide wire was placed and the needle withdrawn. Next, a dilator with an overlying peel away sheath was advanced over the guide wire (Figure1). The wire and dilator were removed, allowing insertion of a Tenckhoff catheter through the final sheath that was peeled away, with the deep cuff buried in the linea-alba after deep dissection. The catheter was secured by purse string sutures around the deep cuff. With the aid of a tunneling tool, a subcutaneous tunnel to the designated skin exit site was fashioned. It was ensured that the second superficial antimicrobial cuff was placed deep within the subcutaneous tunnel at least $2.5-3 \mathrm{~cm}$ from the exit site. The PD catheter insertion site was closed with nylon suture..
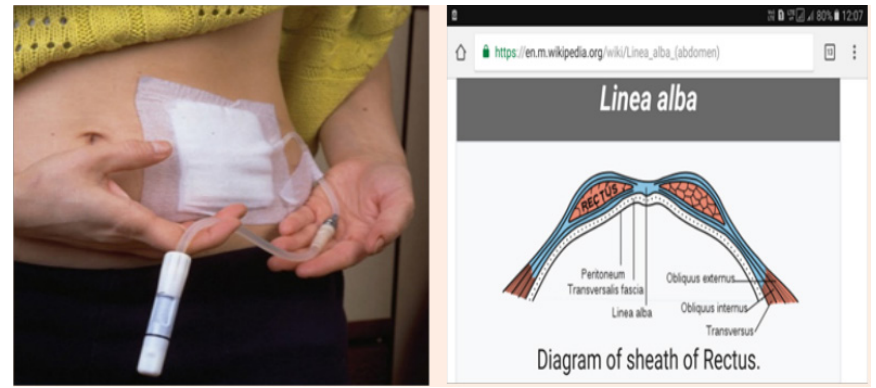

Figure I Catheter insertion.

\section{Modified method for PD catheter insertion}

Initial preparation and infusion of dialysis solution were identical as conventional method. The differences were:-

1. Infraumbilical incision was $1.5-2.0 \mathrm{~cm}$ only

2. Dissection was minimum. 
3. The catheter was not secured by purse-string sutures around the deep cuff.

Rest of the procedure was identical to the conventional method.

\section{Results}

Between Jan 2014 and Dec 2015, a total of 97 catheters were inserted percutaneously using straight and curl Tenckh off catheters ( 66 males, 31 females) by two methods ( 49 by conventional and 48 by modified method; median age $51.65+18.66$ years and $51.79+16.60$ years respectively.

In the conventional group 3 patients developed intra operational bowel injury and requiring prolonged hospitalization but fortunately without mortality. While in the modified group no such complications during the procedure led to any significant patient harm. No patient had significant bleeding and none required blood transfusion (Figures 2-8).

i. Early leak (within 30 days of catheter implantation) was found in $44.89 \%(22 / 49)$ in Conventional method and $4.17 \%(2 / 48)$ in modified method ( $p$ value $<0.05$ ).

ii. Twenty episodes $(41.67 \%)$ of catheter migration were observed in modified method and 3 episodes $(6.122 \%)$ in conventional method ( $p$ value $<0.05$ ) but with significantly better CAPD duration $(21.43+11.69 \mathrm{v} / \mathrm{s} 15.89+9.01)$ and significantly lesser incidences of drainage failure $(8.33 \% \mathrm{v} / \mathrm{s} 46.94 \%)$.

iii. Infection screening during the early period (within 30days) identified $23(46.93 \%)$ episodes in conventional group and $6(12.5 \%)$ episodes after modified percutaneous insertion(p value $<0.05)$

iv. Late onset (after 30 days) peritonitis was also more in the conventional method but with a non-significant trend ( $\mathrm{p}$ value $0.344)$.

The overall median length of hospital stay was $3.62+1.23$ days for modified percutaneous insertions and $4.89+0.99$ days for patients undergoing conventional catheter insertion ( $\mathrm{p}$ value $<0.05$ ).

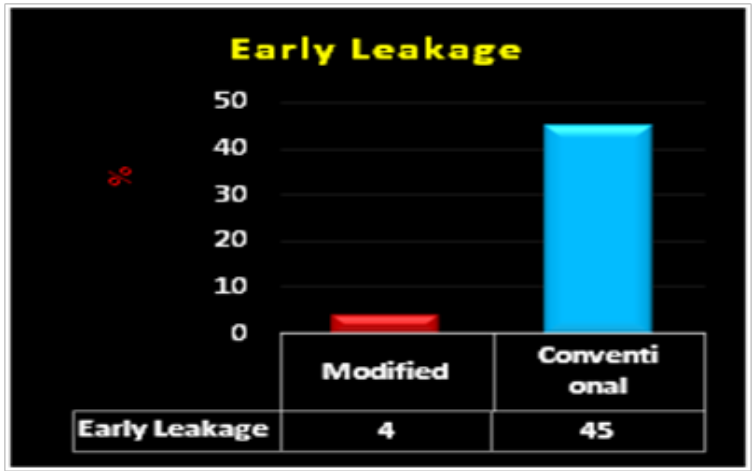

Figure 2 Early Leakage.

\section{Discussion}

This study presents the outcomes following 49 conventional percutaneous (Seldinger) PD catheter insertions and 48 modified insertions within a single centre. This was a prospective study and the main aim was to examine the complication rates and success of conventional and modification of percutaneous insertion, rather than a direct comparison.

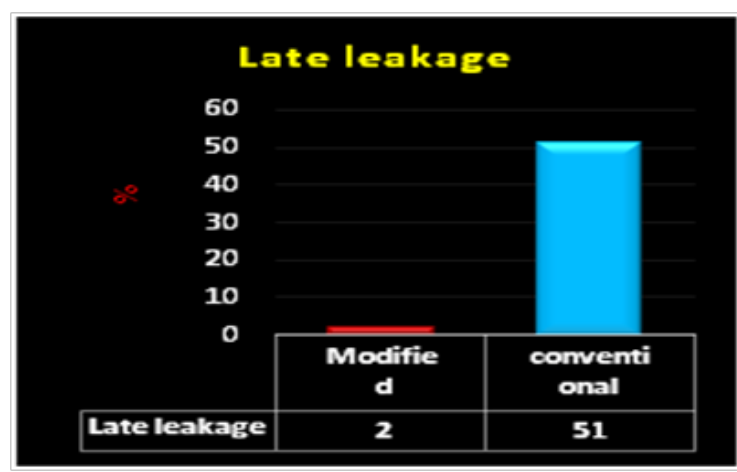

Figure 3 Late leakage.

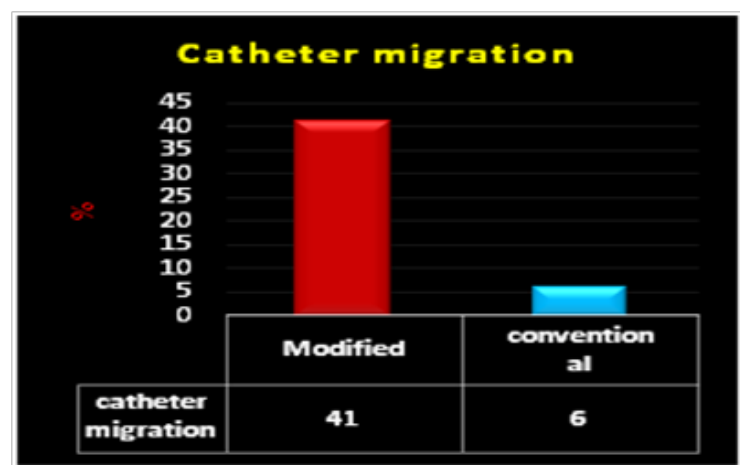

Figure 4 Catheter migration.

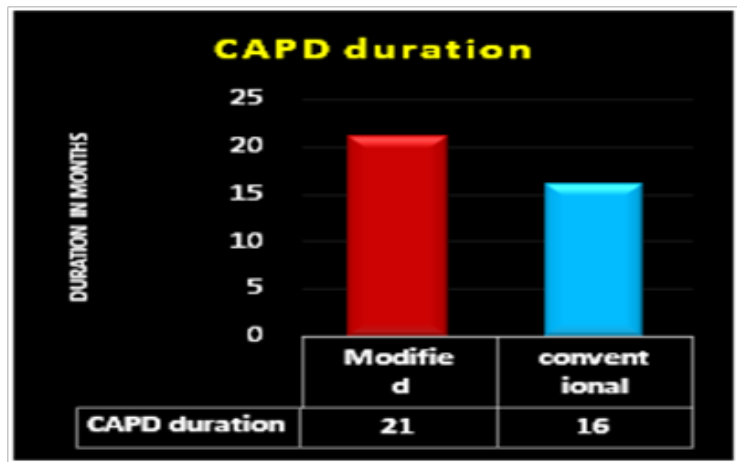

Figure 5 CAPD duration.

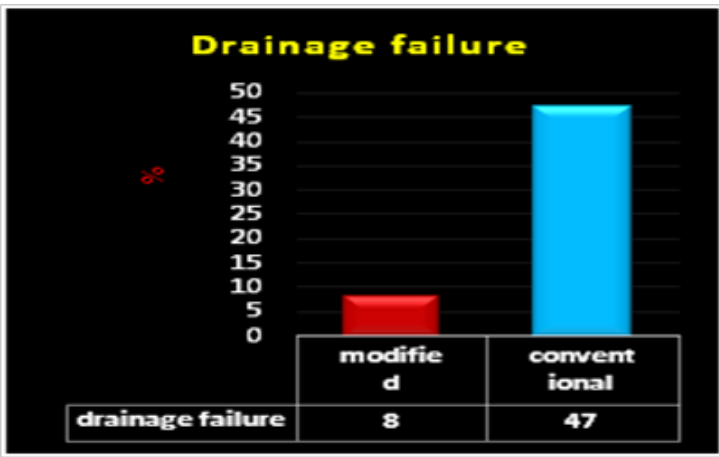

Figure 6 Drainage failure.

In developing countries, the socioeconomic status of patients and the accessibility of the medical system, medical insurance, and equipment have favored CAPD over HD. In certain countries-such as Hong Kong, where PD is the dominant dialysis modality and $80 \%$ of 
the end-stage renal disease population is on PD therapy-PD outcomes appear to be similar to or even better than HD outcomes. ${ }^{10,11}$ Many studies have shown survival advantage for PD over HD in the first 2 years of therapy, with equivalent outcomes to 5 years. ${ }^{12-16}$ Moreover, although overall survival on dialysis therapy has been improving over time, Author dispersed the perception that increasing age, comorbid conditions, and body mass index of the dialysis population are contraindications to PD use. ${ }^{17}$

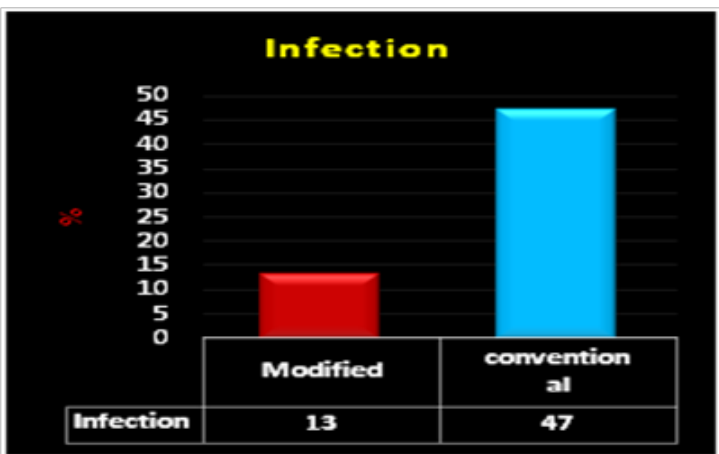

Figure 7 Infection.

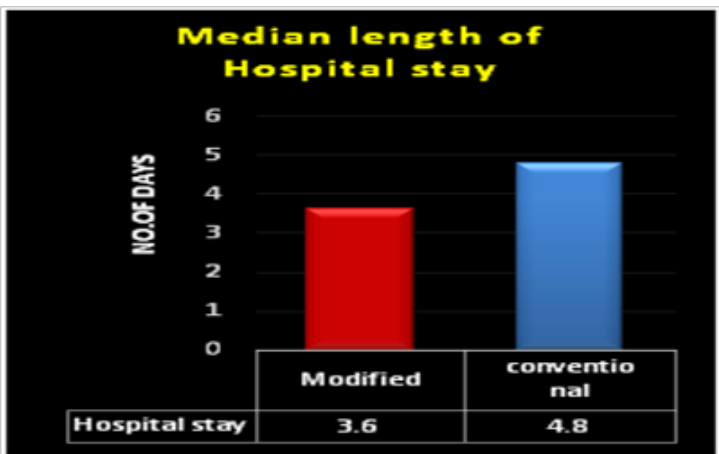

Figure 8 Median length of Hospital stay.

There are several techniques used for the introduction of the PD catheter into the abdominal cavity. Open surgical and laparoscopic techniques are preferred because of their safety and good initial results. The laparoscopic technique is becoming more popular because of its advantage in performing partial omentectomy or adhesiolysis during catheter placement. Percutaneous (radiological) catheter insertion may be less invasive, but bears the risk of unsatisfactory catheter placement and danger of bowel perforation. ${ }^{18}$

As mentioned by Crabtree et al. ${ }^{18}$ best practice for CAPD catheter implantation is to position deep catheter cuff within or below the rectus muscle, but our study found that it required lot of dissection and need for sedation or anesthesia. More time consumed to implant the catheter as well as delay to start CAPD. In Indian setting, patients often present with advanced renal failure requiring immediate dialysis where immediate rapid CAPD cycles just after catheter implant provides relief from uremic complications. The early start of peritoneal dialysis, with break-in periods of shorter than 2 weeks, increasingly has been accepted as an option for patients in need of urgent dialysis. Based on recent studies with a very low incidence of leakage, an early start is acceptable for catheters inserted with technique modifications to ensure a water tight seal around the catheter.,19

\section{Insertion procedure: related complications}

Leakage of PD fluid is a major noninfectious complication reported in percutaneous method, with a reported frequency as high as 12.8
$\% .{ }^{20}$ Whereas in our study modification of implantation technique led to dramatic reduction in incidence of early leak (within 30 days of catheter implantation) to $4.17 \% \mathrm{v} / \mathrm{s} 44.89 \%$ in conventional method as well as late leak (after 30 days of implantation) found in $2.08 \%$ $\mathrm{v} / \mathrm{s} 51.02 \%$ which suggests that minimizing the size of incision and dissection can lead to better outcomes. Conservative means (change to IPD, observation) were applied for the management of late leaks.

Incidences of bowel and urinary bladder injury may occur due to factors like blind insertion methodology, use of trocars, adhesions from previous abdominal surgery, and procedures that include adhesiolysis. ${ }^{21}$ In our study we have encountered only in 3 cases of conventional method $(6.12 \%)$ whereas in modified method it was not observed at all, may be indicative of better learning with more practice.

Drainage failure: Mechanical factors are a major cause of PD catheter malfunction, manifesting as insufficient inflow and/or outflow of dialysate. At the subcutaneous level, kinking of the tubing can lead to slow dialysate flow (including the inflow and outflow rate). Inside the peritoneum, bowel trapping (mostly owing to constipation), omental wrapping, and ensnaring by adhesions, epiploic appendices, or uterine tubes can result in outflow failure (drainage volume being significantly less than the inflow volume). Within the catheter lumen, blood clots or fibrin can lead to slow inflow and outflow rates. In our study outflow problems were seen in 26/49 (53.06 \%) patients in Conventional and $3 / 48(6.25 \%)$ in $\mathrm{M}$ method ( $\mathrm{P}$ value $<0.05)$, even in the presence of more migration episodes but still better PD catheter survival and CAPD duration, suggestive of better methodology while it was reported by various studies between $1.5 \%$ to $34.7 \% .^{22}$ Type of catheter may also influence the better outcomes in our study as curl Catheter was used in modified method which may have led to better catheter survival and lesser drainage problems even with higher episodes of migration.

Peritonitis: In our study episodes of peritonitis were significantly lesser in modified method which may be related to less dissection and procedure time. Modified method was associated with short hospital stays.

Without a prospective randomized controlled trial evaluating insertion technique, it is difficult to ascertain the optimal approach, and the rate of catheter insertion is probably too low in our study for all the conclusions to be appropriately powered. Finally, there may have been a bias that arose during the course of the procedures which was related to the better skills of nephrologists due to ongoing learning. The important principle is that practice makes perfect.

\section{Conclusion}

Results have demonstrated that minimally invasive modified percutaneous catheter insertion has an important role in establishing peritoneal dialysis in a wide range of patients. These results demonstrate that modified percutaneous insertion is associated with very low complication rates, higher long-term catheter patency and without the need of operation theatre and general anesthesia. When timely access to the operating room for laparoscopic catheter implantation or surgery is a rate-limiting step for initiating peritoneal dialysis, catheter insertion by the modified approach represents a reasonable option. We suggest that this technique should be more widely used, as our results reinforce the safe, uncomplicated and welltolerated profile of this cost-effective insertion technique.

\section{Acknowledgements}

None. 


\section{Conflicts of interest}

None.

\section{References}

1. Rottembourg J, Issad B, Allouache M, et al. Recovery of renal function in patients treated by CAPD. Adv Perit Dial. 1989;5: 63-66.

2. Chandna SM, Farrington K. Residual renal function: considerations on its importance and preservation in dialysis patients. Semin Dial. 2004;17(3):196-201.

3. Rottembourg J. Residual renal function and recovery of renal function in patients treated by CAPD. Kidney Int Suppl. 1993;40: S106-110.

4. Crabtree JH, Chow km. Peritoneal dialysis catheter insertion. Seminars in Nephrology. 2017;37(1):17-29.

5. Ozener C, Bihorac A, Akoglu E. Technical survival of CAPD catheters: comparison between percutaneous and conventional surgical placement techniques. Nephrol Dial Transplant. 2001;16:1893-1899.

6. Henderson S, Brown E, Levy J. Safety and efficacy of percutaneous insertion of peritoneal dialysis catheters under sedation and local anaesthetic. Nephrol Dial Transplant. 2009;24(11):3499-3504.

7. Asif A, Byers P, Gadalean F, et al. Peritoneal dialysis underutilization: the impact of an interventional nephrology peritoneal dialysis access program. Semin Dial. 2003;16(3):266-271.

8. Allon M, Soucie JM, Macon EJ. Complications with permanent peritoneal dialysis catheters: experience with 154 percutaneously placed catheters. Nephron. 1988;48:8-11.

9. Jo YI, Shin SK, Lee JH, et al. Immediate initiation of CAPD following percutaneous catheter placement without break-in procedure. Perit Dial Int. 2007;27(2):179-183.

10. Yu AW, Chau KF, Ho YW, et al. Development of the "peritoneal dialysis first” model in Hong Kong. Perit Dial Int. 2007;27(suppl 2):S53-S55.

11. Li PK, Szeto CC. Success of the peritoneal dialysis programme in Hong Kong. Nephrol Dial Transplant. 2008;23(5):1475-1478.
12. Vonesh EF, Snyder JJ, Foley RN, et al. The differential impact of risk factors on mortality in hemodialysis and peritoneal dialysis. Kidney Int. 2004;66(6):2398-2401.

13. Heaf JG, Lokkegaard H, Madsen M. Initial survival advantage of peritoneal dialysis relative to hemodialysis. Nephrol Dial Transplant. 2002;17(1):112-117.

14. Fenton SS, Schaubel DE, Desmeules M, et al. Hemodialysis versus peritoneal dialysis: a comparison of adjusted mortality rates. Am J Kidney Dis. 1997;30(3):334-342.

15. Jaar BG, Coresh J, Plantinga LC, et al. Comparing the risk for death with peritoneal dialysis and hemodialysis in a national cohort of patients with chronic kidney disease. Ann Intern Med. 2005;143(3):174-183.

16. Weinhandl ED, Foley RN, Gilbertson DT, et al.Propensity-matched mortality comparison of incident hemodialysis and peritoneal dialysis patients. J Am Soc Nephrol. 2010;21(3):499-506.

17. Mehrotra R, Kermah D, Fried L, et al. Chronic peritoneal dialysis in the United States: declining utilization despite improving outcomes. $J$ Am Soc Nephrol. 2007;18(10):2781-2788.

18. Crabtree JH. Peritoneal dialysis catheter implantation: avoiding problems and optimizing outcomes. Semin Dial. 2005;28(1):12-15.

19. Sharma AP, Mandhani A, Daniel SP, et al. Shorter break-in period is a viable option with tighter PD catheter securing during the insertion. Nephrology (Carlton). 2008;13(8):672-676.

20. Haggerty S, Roth S, Walsh D, et al. Guidelines for laparoscopic peritoneal dialysis access surgery. Surg Endosc. 2014;28(11):3016-3045.

21. Asif A, Byers P, Vieira CF, et al. Peritoneoscopic placement of peritoneal dialysis catheter and bowel perforation: experience of an interventional nephrology program. Am J Kidney Dis. 2003;42(6):1270-1274.

22. Philip Kam-tao Li, Kai Ming Chow. Importance of peritoneal dialysis catheter insertion by nephrologists: practice makes perfect. Nephrol Dial Transplant. 2009;24(11):3274-3276. 